\title{
AGUDEZA Y MADURACIÓN VISUAL EN LOS NIÑOS Y NIÑAS DEL BARRIO TRES OMBÚES, ESCUELAS 276, 148 Y MONTSERRAT 2008, 2009 Y 2010, MONTEVIDEO, URUGUAY
}

\author{
VISUAL ACUITY AND MATURATION IN \\ CHILDREN FROM TRES OMBÚES NEIGH- \\ BORHOOD, SCHOOLS 276, 148 AND MON- \\ SERRAT; YEARS 2008, 2009 AND 2010, MON- \\ TEVIDEO, URUGUAY
}

ACUIDADE E MATURIDADE VISUAL EM CRIANÇAS DO BAIRRO "TRES OMBÚES", ESCOLAS 276, 148 E MONTSERRAT 2008, 2009 E 2010, MONTEVIDÉU, URUGUAI

\section{Resumen}

Los problemas visuales no diagnosticados son

Hein A., ${ }^{1}$ Olinisky M., ${ }^{2}$ Vignolo J., ${ }^{3}$

${ }^{1}$ Postgrado en Medicina Familiar y Comunitaria. Uruguay

${ }^{2}$ Prof. Adj. De Medicina Familiar y Comunitaria. Uruguay

${ }^{3}$ Prof. Titular de Medicina Familiar y Comunitaria. Uruguay

Trabajo recibido: 15 de diciembre de 2015. Aprobado: 27 de abril de frecuentes en la edad escolar. Un registro de agudeza visual que no llega a la unidad puede corresponder a un hecho mórbido o a un momento particular en el desarrollo de la visión del niño. Es por tanto de interés conocer la edad en la cual se suele completar el proceso de maduración visual. El trabajo realizado describe estos hechos en una población de niños de edad escolar. Objetivo: El presente estudio tiene como objetivo determinar la prevalencia de la agudeza y maduración visual de los niños y niñas de las escuelas 276, 148 y Montserrat, Barrio Tres Ombúes, años 2008, 2009 y 2010.

Material y métodos: Estudio descriptivo transversal de 2088 registros de Agudeza Visual (ojos por separado) correspondientes a 1045 niños entre 5 y 14 años de edad. Los registros se realizaron con cartel Snellen a 5 metros y fueron distribuidos según edad y AV alcanzada. 
Resultados: De los 2088 registros visuales analizados, el 76\% presenta AV 10/10 y el $24 \%$ presenta un registro menor. Al comparar por edad, se observa que la prevalencia del registro AV 10/10 aumenta hasta los 7 años llegando al 86\% y se mantiene entre un 78 y un $87 \%$ hasta los 14 años. Conclusiones: Se puede observar que hasta los 7 años crece el número de ojos que alcanzan el registro 10/10 afirmando la hipótesis de que los niños culminan el proceso de desarrollo y maduración de la visión a esa edad. Encontramos un número importante de registros visuales que no alcanzan AV 10/10 inclusive después de la edad en que los niños suelen haber completado el desarrollo de la AV. Recomendaciones: Realizar Control sistemático de la AV de los niños desde la edad preescolar y realizar la devolución de los resultados a familiares de los niños y docentes.

Palabras clave: niño, desarrollo visual, agudeza visual 10/10

\section{Abstract:}

Undiagnosed visual problems are frequent in school age children. A record of visual acuity not reaching the unit may correspond to a morbid fact or to a particular moment in the development of the childss sight. So, it is interesting to know the age when the maturation of the visual process usually finishes. This work describes these facts in a population of school age children. Objective: The objective of this study is to determine the prevalence of visual acuity and maturation in children from schools 276, 148 and Montserrat, in Tres Ombúes neighborhood, years 2008, 2009 and 2010. Material and Methods: Cross sectional descriptive study of 2088 records of VA (separate eyes) corresponding to 1045 children between 5 and 14 years old. Records were taken using Snellen chart at 5 meters and were distributed according to age and VA reached. Results: From the 2088 visual records that were analyzed, 76\% presented VA 10/10 and 24\% presented a lower record. When comparing according to age, we observe that the prevalence of the record VA 10/10 increases up to age 7 reaching $86 \%$ and remains between 78 and $87 \%$ up to age 14. Conclusions: We can observe that up to age 7 , the number of eyes reaching the record 10/10 increases, so we can confirm the hypothesis that children complete the process of visual development and at that age. We find an important number of visual records which do not reach VA 10/10 even after the age when children have usually completed VA development. Recommendations: Carry out systematic VA control of pre-school age children and provide feedback to children >s relatives and teachers.

Key Works: child, visual development, visual acuity 10/10

\section{Resumo:}

Os problemas de visão não diagnosticados são frequentes na idade escolar. Um registro da acuidade visual que não atinge a unidade pode se corresponder com um evento mórbido ou um momento particular no desenvolvimento da visão da criança. É, portanto, de interesse conhecer a idade na qual é completado o processo de maturidade visual. O trabalho desenvolvido descreve esses eventos em uma população de crianças em idade escolar. Objetivo: Este estudo tem como alvo determinar a prevalência de acuidade e maturidade visual de crianças das escolas 276, 148 e Montserrat, Bairro Três Ombúes, anos de 2008, 2009 e 2010. Material e Métodos: Estudo transversal, de 2.088 registros de AV (olhos separadamente) correspondentes a 1045 crianças entre 5 e 14 anos de idade. Os registros foram feitos com o cartaz Snellen a 5 metros e foram distribuídos por idade e AV atingido. Resultados: Dos 2088 registros visuais analisados, 76\% têm AV 10/10, e 24\% apresentam um registro inferior. Ao compararmos por idade, observa-se que a prevalência 
do registro AV 10/10 aumenta até os 7 anos de idade, atingindo 86\% e permanece entre 78 e $87 \%$ até os 14 anos de idade. Conclusões: Pode-se deduzir que até 7 anos um número crescente de olhos atinge o registro 10/10 confirmando a hipótese de que as crianças culminam o processo de desenvolvimento e maturidade da visão nessa idade. Achamos um número significativo de registros visuais que não atingem AV 10/10, mesmo após a idade em que as crianças costumam ter concluído o desenvolvimento de AV. Recomendações: Controlar sistematicamente a AV de crianças desde a idade pré-escolar e fazer o retorno de resultados aos parentes das crianças e professores.

Palavras-chave: criança, desenvolvimento visual, acuidade visual 10/10

\section{Introducción.}

La edad preescolar y primeros años de la edad escolar coincide con la etapa de la vida en la que debe completarse el desarrollo de la AV. Un número significativo de niños nunca alcanza una AV plena (10/10) para ambos ojos y otros instalan un déficit de AV en estas etapas. En muchos casos ni el niño ni el docente ni su entorno familiar se percata de dicho déficit a menos que se realice un control de AV de ambos ojos por separado. Dado que este período de la vida coincide con la existencia de una plasticidad cerebral remanente es también un período de oportunidad para intentar corregir el déficit y lograr el máximo desarrollo visual posible. Se ha propuesto el cribado de la AV visual en la edad preescolar y primeros años de la escolar como una línea de acción para detección precoz de patología y tratamiento oportuno [1]. El diagnóstico de la patología ocular es resorte del oftalmólogo, de todas maneras un registro de AV menor al esperado en cualquiera de los ojos constituye un signo de alarma para referir al niño al especialista para su evaluación. La medida de la agudeza visual es un instrumento primordial para describir el estado de la función visual de la persona y puede ser determinada con técnicas simples y de bajo costo a partir de la etapa verbal del niño [2]. La determinación de la AV en el niño tiene algunas particularidades en cuanto a técnica, así como también la interpretación de los resultados cuya norma está en función de la edad y el desarrollo de la persona. Cuando se encuentra un registro de AV que no llega a la unidad, en un niño preescolar o primeros años de la escuela, se plantea la pregunta si se está ante una alteración o si se está ante un momento particular de su desarrollo y maduración. Desde un punto de vista poblacional un porcentaje importante de niños no alcanza la unidad o AV 10/10. La edad en la que culmina el desarrollo de la AV marcaría un hito a la hora de interpretar este hecho. En la población que no llega a la AV 10/10 y es menor a dicha edad podemos considerar factores fisiológicos como falta de maduración y esperar un proceso evolutivo hacia la adquisición de registros mejores, mientras que después de dicha edad la incidencia de dicho factor es poco probable. Esto en todo caso no debe ir en detrimento de la responsabilidad de descartar patología para cada caso en particular en cualquiera de los dos grupos.

¿A qué edad alcanza la madurez el desarrollo visual del niño? Según revisión de literatura: La agudeza visual normal debe desarrollarse mediante exposición de cada retina a una imagen focalizada desde el nacimiento hasta los 6-9 años de edad [3]. El desarrollo de la visión del niño es un fenómeno ininterrumpido. Ya a las 30 semanas de gestación se puede ver el cierre palpebral en respuesta a la luz brillante. Al nacimiento aparece la fijación visual, cuyo desarrollo se completa a los 2 meses. A los 3 meses se completa el desarrollo del seguimiento visual y a los 4 meses la acomodación. La agudeza visual con mirada preferencial y a caracteres del cartelón de Snellen propia del adulto se comienza a ver a partir de los 2 años. Las técnicas complementarias sugieren AV 1/20 al nacimiento, 
4/10 al año, pudiendo alcanzar a 10/10 a los 2 años. A los 6 meses ya se puede comprobar estereopsia (visión tridimensional), que mejora hasta los 7 años de vida [4]. La capacidad resolutiva varía con la edad. A los 4 años por ejemplo la AV media es de 0,8 con una dispersión (+- dos desviaciones estándar) de 1 a 0,6. A los 6 a 8 años los ojos emétropes alcanzan la unidad [5]. Sabemos que la visión se va desarrollando en forma progresiva a partir del nacimiento. Se alcanza la visión 10/10 (leer 10 líneas en 10 en el cartel de Snellen) a los 5 a 6 años. Muchos niños alcanzan la visión 10/10 a edades mayores (6).

El presente estudio es un análisis de los registros de AV con el objetivo general de determinar la prevalencia de agudeza y maduración visual en la población de los niños y niñas citados.

Los objetivos específicos son cuantificar y caracterizar el déficit de AV y visualizar el logro de la AV 10.10. Otro objetivo es el de comparar los resultados con un estudio en una población similar en otro país latinoamericano. Para ello se debe establecer la AV 1-10 en los niños y niñas de 5-14 años, se determina la AV 10.10 y se establece AV según edad.

\section{Material y métodos}

Se realizó un estudio descriptivo transversal que analiza un registro de AV efectuado con cartel de Snellen a 5 metros en una población de niños entre 5 y 14 años, de las escuelas 276, 148 y Montserrat del barrio Tres Ombúes en los años 2008, 2009, 2010. El barrio Tres Ombúes pertenece a la sección policial 9vna. CCZ 14, Área censal 12 de Montevideo, Uruguay.

\section{Procedimientos:}

Los Alumnos pasaron al salón de evaluación en grupos de a tres siendo recibidos por la maestra auxiliar de la escuela. De esta manera se logró mitigar la timidez y el temor, favoreciendo la colaboración. A su vez se logró mantener el grado de silencio y concentración necesario para la prueba. Luego de anotar el nombre (tomado de la lista de asistencia diaria de la escuela confeccionado por clase, sexo, edad), se pasó a realizar un breve examen ocular general incluyendo el test de Hirschberg. Se realizó la lectura de la agudeza visual con cartel de Snellen a $5 \mathrm{~m}$, primero el ojo derecho luego el ojo izquierdo cubriendo respectivamente el ojo contralateral con un oclusor y se registró el resultado. Se puso especial cuidado en una iluminación uniforme con una lámpara de $60 \mathrm{~W}$ enfocada sobre el cartel desde un sitio lateral. En caso de percibir dificultad para la interpretación de los números se realizó la prueba usando los Optotipos «E» desde la línea del optotipo mas grande a la más pequeña. Se registró el valor correspondiente a la línea de menor tamaño que alcanzó a leer en forma correcta. Para la evaluación de resultados se usó planilla de EXCEL.

Se estudiaron 1063 niños; 536 del sexo femenino (50,42\%) y 527 del sexo masculino (49,58\%). Los alumnos que presentaron deficiencia de AV y factores ambliogénicos fueron referidos a oftalmólogo para su evaluación a fin de optimizar su salud visual. A efecto de analizar los registros de AV se mantuvo en completo anonimato a todos los participantes. Dados los objetivos planteados se excluyeron del análisis los registros de los 18 niños con AV corregida por lentes. 2 niños presentaban visión monocular. Los 1045 niños que integran el análisis corresponden a 2088 registros de AV realizados en 2088 ojos. Los registros visuales de los ojos fueron distribuidos según: edad y agudeza visual según líneas del cartel de Snellen alcanzadas, 1-2 líneas, 3-5 líneas,6, 7, 8, 9 y 10 en 10 líneas, (AV 10/10).Para cada edad se determinó el número de ojos que alcanzaron cada 
registro consignando frecuencia absoluta y relativa. Luego se comparó la prevalencia de la AV 10/10 según edad. Finalmente se compararon los resultados con un estudio en una población de características similares realizado en Cuba en 2011.

\section{Resultados y discusión}

Tabla 1: Distribución de los registros visuales (ojos por separado) de los niños y niñas según AV y edad, escuelas 276, 148 y Montserrat, Barrio Tres Ombúes, Años 2008, 2009 y 2010.

\begin{tabular}{|c|r|r|r|r|r|r|r|r|r|r|r|r|r|r|r|r|}
\hline Agud & \multicolumn{2}{|c|}{$\mathbf{5}$ años } & \multicolumn{2}{|c|}{$\mathbf{6}$ años } & \multicolumn{2}{|c|}{$\mathbf{7}$ años } & \multicolumn{2}{|c|}{$\mathbf{8}$ años } & \multicolumn{2}{|c|}{$\mathbf{9}$ años } & \multicolumn{2}{|c|}{$\mathbf{1 0}$ años } & \multicolumn{2}{|c|}{$\mathbf{1 1}$ años } & \multicolumn{2}{|c|}{$\mathbf{2 - 1 4}$ años } \\
\hline Visua & FA & FR\% & FA & FR\% & FA & FR\% & FA & FR\% & FA & FR\% & FA & FR\% & FA & FR\% & FA & FR\% \\
\hline $\mathbf{1 0 2}$ & 1 & 0,29 & 1 & 0,30 & 0 & 0,00 & 0 & 0,00 & 0 & 0,00 & 5 & 2,18 & 5 & 2,16 & 4 & 1,61 \\
\hline $\mathbf{3 a 5}$ & 10 & 2,94 & 8 & 2,40 & 7 & 3,13 & 5 & 1,64 & 2 & 1,12 & 5 & 2,18 & 6 & 2,60 & 12 & 4,84 \\
\hline $\mathbf{6}$ & 6 & 1,76 & 8 & 2,40 & 4 & 1,79 & 2 & 0,66 & 7 & 3,93 & 2 & 0,87 & 5 & 2,16 & 4 & 1,61 \\
\hline $\mathbf{7}$ & 31 & 9,12 & 17 & 5,09 & 8 & 3,57 & 8 & 2,63 & 13 & 7,30 & 5 & 2,18 & 8 & 3,46 & 6 & 2,42 \\
\hline $\mathbf{8}$ & 46 & 13,53 & 23 & 6,89 & 7 & 3,13 & 3 & 0,99 & 5 & 2,81 & 3 & 1,31 & 3 & 1,30 & 4 & 1,61 \\
\hline $\mathbf{9}$ & 73 & 21,47 & 50 & 14,97 & 5 & 2,23 & 21 & 6,91 & 11 & 6,18 & 13 & 5,68 & 15 & 6,49 & 9 & 3,63 \\
\hline $\mathbf{1 0}$ & 173 & 50,88 & 227 & 67,96 & 193 & 86,16 & 265 & 87,17 & 140 & 78,65 & 196 & 85,59 & 189 & 81,82 & 209 & 84,27 \\
\hline total & 340 & 100,00 & 334 & 100,00 & 224 & 100,00 & 304 & 100,00 & 178 & 100,00 & 229 & 100,00 & 231 & 100,00 & 248 & 100,00 \\
\hline
\end{tabular}

Se comparó la frecuencia relativa del registro 10/10 para cada edad desde 5 a 11 y 12-14 años respectivamente.

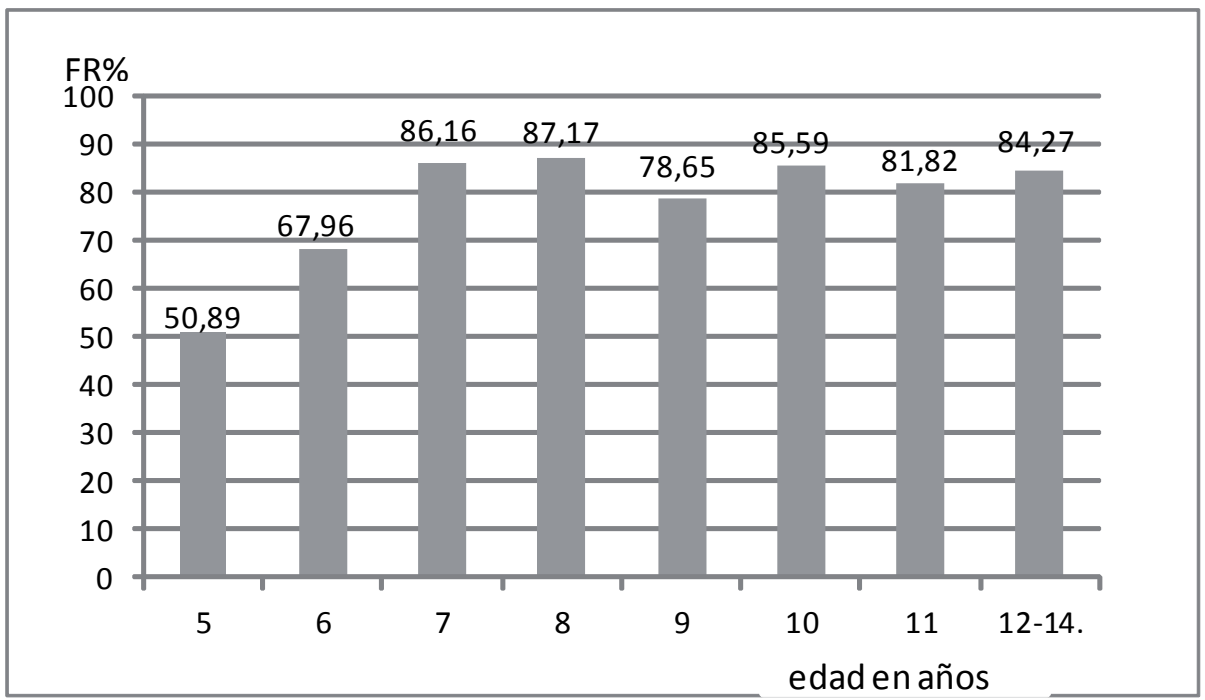

Gráfico 1: Distribución de los registros visuales (ojos por separado) de los niños y niñas según la AV 10/10 y edad, escuelas 276, 148 y Montserrat, Barrio Tres Ombúes, 2008, 2009 y 2010.

Según la tabla 1 se observa que el registro de AV 10/10 se encuentra para el 76\% de los ojos examinados. La FR del registro de AV 10/10 a los 5 años fue alcanzada en el 50,9 \%; a los 6 años en el 68\% y a los 7 años 86,2\% y luego oscila entre un 87 y $72 \%$ manteniéndose 
en un promedio de $84 \%$ de los ojos examinados hasta los 14 años. En la representación gráfica de la figura 1 se observa que hasta los 7 años aumenta el contingente de ojos que logra el registro de AV 10/10 y luego se mantiene alrededor del 84\%. En términos gráficos habría un punto de inflexión a los 7 años.

Por otra parte se destaca que ninguna edad alcanza una FR del 100\% para la AV 10/10. A partir de los 7 años y para cada edad hay un porcentaje de ojos que no alcanzan dicho valor y que oscila entre el 13 y 22\%. Esto condice con varios estudios realizados que afirma que 1 de cada 5 escolares estudiados presenta defectos de refracción. [5-7] En un trabajo realizado en Buenos Aires, Argentina, que toma como referencia que la AV esperada para edad de 7 años es 10/10, encuentra que un 24\% de los niños de 7 años y mayor no alcanzan dicho valor [8]. Se observa en este estudio que en los menores de 7 años la FR de los ojos que no alcanzan AV 10/10 es aún mayor, dado que muchos aún no han completado el proceso fisiológico del desarrollo. Para afirmar estos conceptos se despliegan los resultados según la siguiente gráfica.

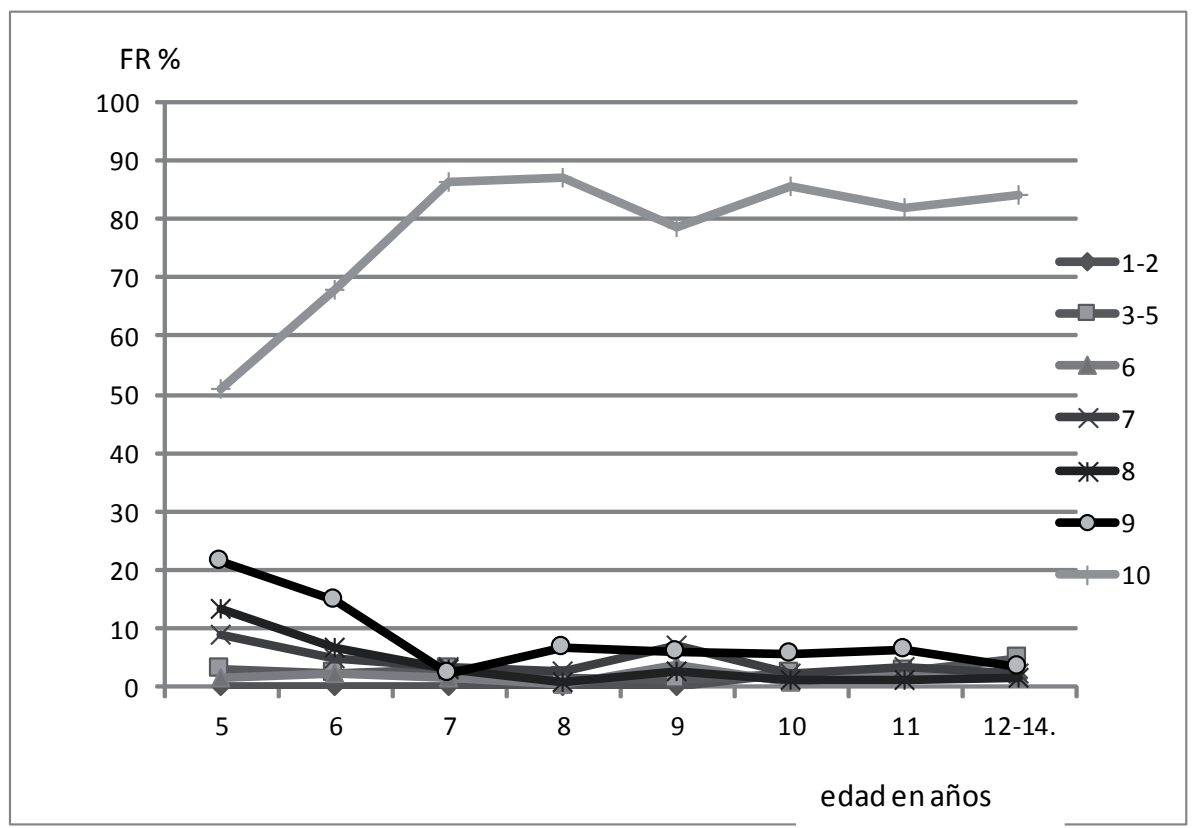

Gráfico 2: Distribución de los registros visuales (ojos por separado) de los niños y niñas de 5-14 años, según la AV alcanzada, escuelas 276, 148 y Montserrat, Barrio Tres Ombúes, años 2008, 2009 y 2010

La distribución de las FR de los ojos examinados según AV alcanzada en el gráfico 2 muestra que los valores que representan el déficit de AV más importante como 1-2, 3-5 y 6 líneas, están representados por un porcentaje acumulado menor presente en todas las edades, que oscila entre 2,3\% y 5,1\%; mientras que el déficit leve con valores de 7, 8 y 9 líneas se observa fundamentalmente en los menores de 7 años. La prevalencia 10/10 crece hasta los 7 años y lo hace a expensas de los registros de déficit de AV leve 9/10, 8/10 y $7 / 10$.

En Setiembre de 2010 a febrero 2011 se realizó un registro de AV en niños y niñas de 5 a 12 años de edad, alumnos de la primaria Pedro Murillo, en el municipio de Marianao, Ciudad de Habana, Cuba [9]. Se examinaron 422 niños registrando la AV en 844 ojos. Los resultados de los registros de AV fueron desplegados por ojos examinados 
y se distribuyeron en 4 grupos según el grado de AV alcanzada. Se acumularon a los que alcanzaron a leer entre 9 y 10 líneas, valores que fueron considerados unidad, 6-8 líneas, 3-5 líneas y 1-2 líneas. Estos últimos 3 grupos fueron referidos a oftalmólogo para valorar posibles trastornos de refracción y descartar ambliopía. A efectos de comparar los resultados y agrupando de forma similar los resultados de este estudio se encuentra que el 85,5\% de los registros alcanzó 9 o 10 líneas (80,6\% en el estudio de Cuba); 11\% de los registros de este estudio alcanzó 6-8 líneas (12,6\% en el estudio de Cuba); el 2,7\% registra 3-5 líneas (6,8\% en el estudio de Cuba) y el 0,8\% solo alcanzó 1-2 líneas (no hubo registros en el estudio de Cuba). Se puede observar aquí que los estudios son comparables y marcan las mismas tendencias.

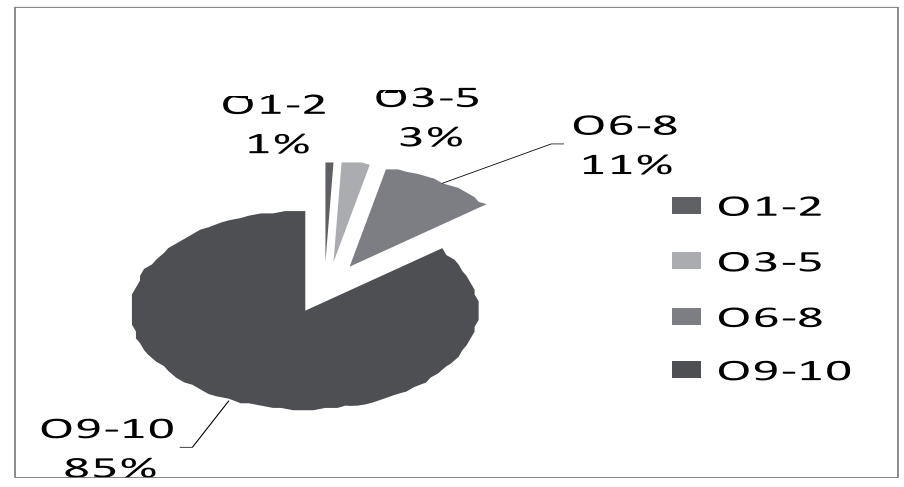

Gráfico 3: Distribución de los registros visuales ojos por separado de los niños y niñas agrupados según la AV alcanzada, escuelas 276, 148 y Montserrat, Barrio Tres Ombúes, Uruguay, años 2008, 2009 y 2010.

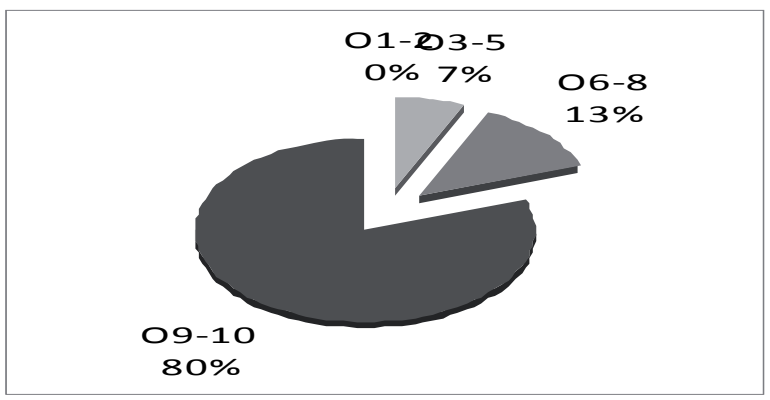

Gráfico 4: Distribución de los registros visuales ojos por separado de los niños y niñas agrupados según la AV alcanzada, primaria Pedro Murillo, en el municipio de Marianao, Ciudad de Habana, Cuba (Fuente: Revista cubana de oftalmología vol 24 n² Ciudad de la Habana jul-dic 2011) (9).

\section{Conclusiones}

El 76\% de los registros de AV (ojos por separado) de los niños de las escuelas 276, 148 y Montserrat del barrio Tres Ombúes estudiados en 2008-2010 presentan AV10/10.

La prevalencia del registro de AV 10/10 aumenta hasta los 7 años llegando al 86\% manteniéndose luego, a edades mayores, en valores que oscilan entre el $78 \%$ y $87 \%$. Esto afirma la hipótesis de que muchos niños completan su desarrollo visual recién a los 7 años. Condice además con el hecho en general aceptado de que en el proceso de maduración postnatal, el período desde el nacimiento a los 2 años es de mayor plasticidad sensorial y de los 2 a 7 u 8 años la misma disminuye y se extingue [7].

Por otra parte, para ninguna edad se llega al 100\% de registros de AV 10/10. El 24\% de 
los registros presenta valores menores a 10/10. Para los niños entre 7 y 14 años de edad ello representaría un déficit visual y deberán ser estudiados para descartar alteraciones de refracción o patología. Para los niños de edad menor a 7 años no haber alcanzado registros de AV 10/10 podrá corresponder a un momento evolutivo de su desarrollo visual (en especial cuando la desviación respecto al registro 10/10 es leve), pasible de seguimiento, o a un déficit visual por lo que también deberá ser valorado para descartar alteraciones de refracción o patología. Si se compara la prevalencia de AV alcanzada en la población estudiada en Tres Ombúes Uruguay con la del barrio Marianao Cuba donde se consideró unidad los registros de 9/10 y 10/10 se observan las mismas tendencias.

\section{Recomendaciones}

Se ha comprobado un importante número de niños con ojos que no logran una AV 10/10 a todas las edades inclusive en aquellos en los que se suele haber completado el desarrollo de la AV. Para lograr una detección precoz de las alteraciones es importante el control sistemático de la visión incluyendo la AV en todos los niños en el primer nivel de atención, desde la edad preescolar ni bien el niño pueda colaborar con los Test para AV. Tomando en cuenta el importante número de niños con alteraciones en sus registros será necesario el trabajo coordinado con el especialista acordando pautas de referencia. Realizar una devolución de resultados a familiares de alumnos y docentes de las escuelas a fin de sensibilizarles en la necesidad del control de AV en el niño a fin de lograr el mejor desarrollo de la AV para cada uno de ellos.

\section{Bibliografía}

1. Andújar E.M., Alvarez-Dardet C., Gil González D., Evidencia científica y recomendaciones sobre cribado de agudeza visual. Revisión Bibliográfica Rev. Esp. Salud Pública 2012; 86: 575-588 Nro. 6 nov-Dic 2012. Disponible en: http://scielo.isciii.es/pdf/resp/v86n6/04_revision_bibliografica.pdf

2. Parra A., Revisión de tema: Tamización de ambliopía en cuidado primario, Universidad de Medicina de Bogotá (Colombia), 50 (2): 225-236, Abril/Junio 2009

3. Omondi L., Nyong’O, Del Monte A., Deficiencia visual en la infancia: función visual normal y anormal en el contexto de la discapacidad en el desarrollo; Clínicas pediátricas de Norteamérica 2008 Volumen 55 Nro. 6, 2008 pág. 1403-1415

4. Kaufman L., Curnyn K, Examen oftalmológico en el consultorio del pediatra, Clínicas pediátricas de Norteamérica, Volumen 1/2003 Oftalmología Pag.25-39; 41-53;203-204;

5. Delgado J., Merino M., Grupo Previnfad/PAPPS Detección de problemas visuales en los niños: cuando y como pag. 13-18

6. Demicheli H. MSP Uruguay 2006 Programa Nacional de salud ocular, pág. 5

7. Velazquez M., Gallareta M., Bravo C. Pesquisa de alteraciones visuales en una población preescolar de Montevideo. Archivos pediat. Uruguay 1995652 pág. 23.2

8. Pastorino N, Penerini Y., Programa de detección de déficit de la agudeza visual en escolares sin patología ocular aparente, Arch Arg. Pedriat. 1998/ Vol. 96: 236. Disponible en: http://www.sap.org.ar/docs/publicaciones/archivosarg/1998/98_236_241.pdf

9. $\quad$ Estévez Miranda, Y., Naranjo Fernández, R.M., Pons Castro, L., Méndez Sánchez, T., Rúa Martínez, R., Defectos refractivos en estudiantes de la Escuela "Pedro D. Murillo”, Instituto Cubano de Oftalmología “Ramón Pando Ferrer”. La Habana, Cuba Rev Cubana Oftalmol vol.24 no.2 Ciudad de la Habana jul.-dic. 2011 Disponible en: http://scielo.sld.cu/pdf/oft/v24n2/oft13211.pdf 